\title{
MENINGITIS POR Streptococcuspneumoniae RESISTENTE A CEFTRIAXONA: REPORTE DE UN CASO
}

\author{
Marcos Saavedra-Velasco 1,2,a,b, Mariel Tapia-Cruz 1,a , J. Antonio Grandez-Urbina 2,3,a, \\ Saarah Zegarra Del Rosario-Alvarado 2,a, Fernando Mendo-Urbina 1,a,b, Rafael Pichardo-Rodriguez 1,2,a
}

\begin{abstract}
RESUMEN
La meningitis infecciosa es una emergencia médica. Dentro del espectro de agentes infecciosos, el más importante es el Streptococcus pneumoniae, agente etiológico más frecuente de la meningitis bacteriana. El inicio de tratamiento antimicrobiano empírico es de gran importancia y considera a las cefalosporinas de tercera generación como la primera alternativa. Sin embargo, casos de resistencia a ceftriaxona han sido reportados en diversas partes del mundo, siendo un problema emergente, por lo que necesita una reconsideración de los esquemas antibióticos empíricos actuales. Presentamos el caso de un varón de 56 años que presenta meningitis aguda infecciosa por Streptococcus pneumoniae resistente a ceftriaxona, que respondió favorablemente al tratamiento empírico combinado con ceftriaxona y vancomicina y que durante su estadía hospitalaria se detectó la presencia de hipotiroidismo y megacisterna magna.
\end{abstract}

Palabras claves: Meningitis Nuemocócica; Ceftriaxona; Farmacorresistencia Microbiana. (fuente: DeCS BIREME).

\section{CEFTRIAXONE-RESISTANT Streptococcus pneumoniae MENINGITIS: CASE REPORT}

\begin{abstract}
Infectious meningitis is a medical emergency. Within the spectrum of infectious agents, the most important is Streptococcus pneumoniae, the most frequent etiological agent of bacterial meningitis. The initiation of empirical antimicrobial treatment bears great importance and considers third-generation cephalosporins as the first alternative. However, cases of ceftriaxone resistance have been reported in several regions of the world. This has become an emerging problem in need of reconsideration of the current empirical antibiotic treatment schemes. We present the case of a 56-year old man with acute infectious meningitis caused by ceftriaxone-resistant Streptococcus pneumoniae, who responded favorably to combined empirical treatment with ceftriaxone and vancomycin and to whom, during his hospital stay, the presence of hypothyroidism and mega cisterna magna was diagnosed.
\end{abstract}

Keywords: Pneumococcal meningitis, Ceftriaxone, Microbial Drug Resistance (source: MeSH NLM).

\section{INTRODUCCIÓN}

La meningitis infecciosa representa una emergencia médica, relacionándose directamente el pronóstico del paciente con el tratamiento precoz, afectando directamente la morbi-mortalidad del mismo ${ }^{(1)}$. El agente etiológico más frecuente de la meningitis bacteriana es el Streptococcus pneumoniae (SP) ${ }^{(1)}$.

El inicio de tratamiento antimicrobiano empírico considera a las cefalosporinas de tercera generación como primera alternativa terapéutica; sin embargo, en combinación con otros antibióticos como vancomicina en adultos, es aún controversial, esto debido a la escasez de estudios de resistencia a ceftriaxona (RC) en este grupo etáreo (2). No obstante, casos de RC están siendo reportados en la actualidad en diversas partes del mundo, siendo un problema emergente, por lo que se necesita reconsideración de los esquemas terapéuticos empíricos actuales para la población adulta ${ }^{(2-4)}$.

Por otro lado, el hipotiroidismo se relaciona con una disminución del conteo de linfocitos y de la respuesta inmunitaria, asociándose probablemente a una mayor

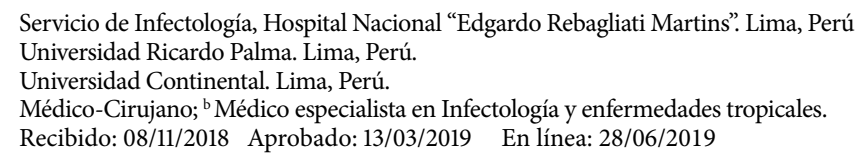

Citar como: Saavedra-Velasco M, Tapia-Cruz M, Grandez-Urbina JA, Zegarra Del Rosario-Alvarado S, Mendo-Urbina F, Pichardo-Rodriguez R. Meningitis por Streptococcus pneumoniae resistente a ceftriaxona: reporte de un caso. Rev Peru Med Exp Salud Publica. 2019;36(2):349-52. doi: http://dx.doi.org/10.17843/ rpmesp.2019.362.4036. 
susceptibilidad a procesos infecciosos ${ }^{(5)}$. La megacisterna magna (MM) es una malformación congénita de la fosa posterior, con un posible efecto sobre la circulación del líquido céfalo-raquídeo (LCR) ${ }^{(6)}$.

Presentamos el caso de un varón con hipotiroidismo y MM que presentó meningitis aguda infecciosa por SP con $\mathrm{RC}$, que respondió favorablemente al tratamiento empírico combinado con ceftriaxona y vancomicina.

\section{REPORTE DE CASO}

Varón de 56 años, procedente de Lima, Perú con antecedentes de cirugía en brazo izquierdo por retiro de proyectil de arma de fuego hace cinco años, y colecistectomía por pólipos vesiculares, sin antecedentes médicos de importancia.

En junio de 2018 ingresa a un hospital de la seguridad social de Lima donde presenta alteración de la conducta, desorientación y sensación de alza térmica. Familiar refiere que paciente presentó otalgia días previos al inicio del cuadro, sin otorrea, con manejo sintomático. Al examen físico se encuentra sequedad de piel y caída de cabello, en el examen neurológico, hay un puntaje de la escala Glasgow de 11/15, signos meníngeos (Brudzinky y Kerning positivos), desorientación en tiempo y agitación psicomotriz. En el hemograma hay leucocitosis (21 $\left.850 \mathrm{cel} / \mathrm{mm}^{3}\right)$, con $94,3 \%$ de neutrófilos y proteína $C$ reactiva (PCR) en $38,4 \mathrm{mg} / \mathrm{dL}$. La radiografía de tórax sin cambios aparentes.

Ante la sospecha de una evidente afectación meníngea, se le realizó una punción lumbar con los siguientes resultados:
LCR de aspecto amarillento y turbio, con celularidad de 850 leucocitos $/ \mathrm{mm}^{3}$ a predominio de polimorfonucleares (83\%), glucosa en $51 \mathrm{mg} / \mathrm{dL}$, y proteínas en $741 \mathrm{mg} / \mathrm{dL}$. Los niveles de Adenosina Desaminasa (ADA) se encontraban en $27,8 \mathrm{U} / \mathrm{L}$. Se obtuvo un cultivo positivo para SP a la espera del resultado del antibiograma, confirmándose el diagnóstico de meningitis aguda infecciosa.

En la tomografía cerebral sin contraste y resonancia magnética se observa en la fosa posterior una imagen compatible con MM como variante anatómica y ausencia de signos de hipertensión endocraneana (Figura 1).

Se inició antibioticoterapia empírica con ceftriaxona $2 \mathrm{gr}$. endovenoso (EV) cada 12 horas, vancomicina $1 \mathrm{gr}$. EV cada 12 horas y dexametasona $4 \mathrm{mg}$. EV cada 6 horas. A las 48 horas se evidenció mejoría del sensorio.

Se solicita un hemograma control donde se evidencia leucocitosis $\left(11730 \mathrm{cel} / \mathrm{mm}^{3}\right)$, conteo de linfocitos absoluto en $820\left(\mathrm{cel} / \mathrm{mm}^{3}\right)$ y PCR en $3,8 \mathrm{mg} / \mathrm{dL}$.

Se encuentran lesiones vesiculares con borde eritematoso, dolorosas a la palpación en comisura labial izquierda y en paladar duro asociadas a fiebre. Se solicita un hemograma control donde se evidencia leucocitosis $\left(11730 \mathrm{cel} / \mathrm{mm}^{3}\right)$, conteo de linfocitos absoluto en $820\left(\mathrm{cel} / \mathrm{mm}^{3}\right)$ y PCR en $3,8 \mathrm{mg} / \mathrm{dL}$.

Durante la visita médica se constata la persistencia de caída de cabello y sequedad de piel, motivo por el que se solicita un perfil tiroideo, el cual muestra hormona estimulante de tiroides en 55,8 Ul/ml, T3 libre en 2,51 ng/dL y T4 libre

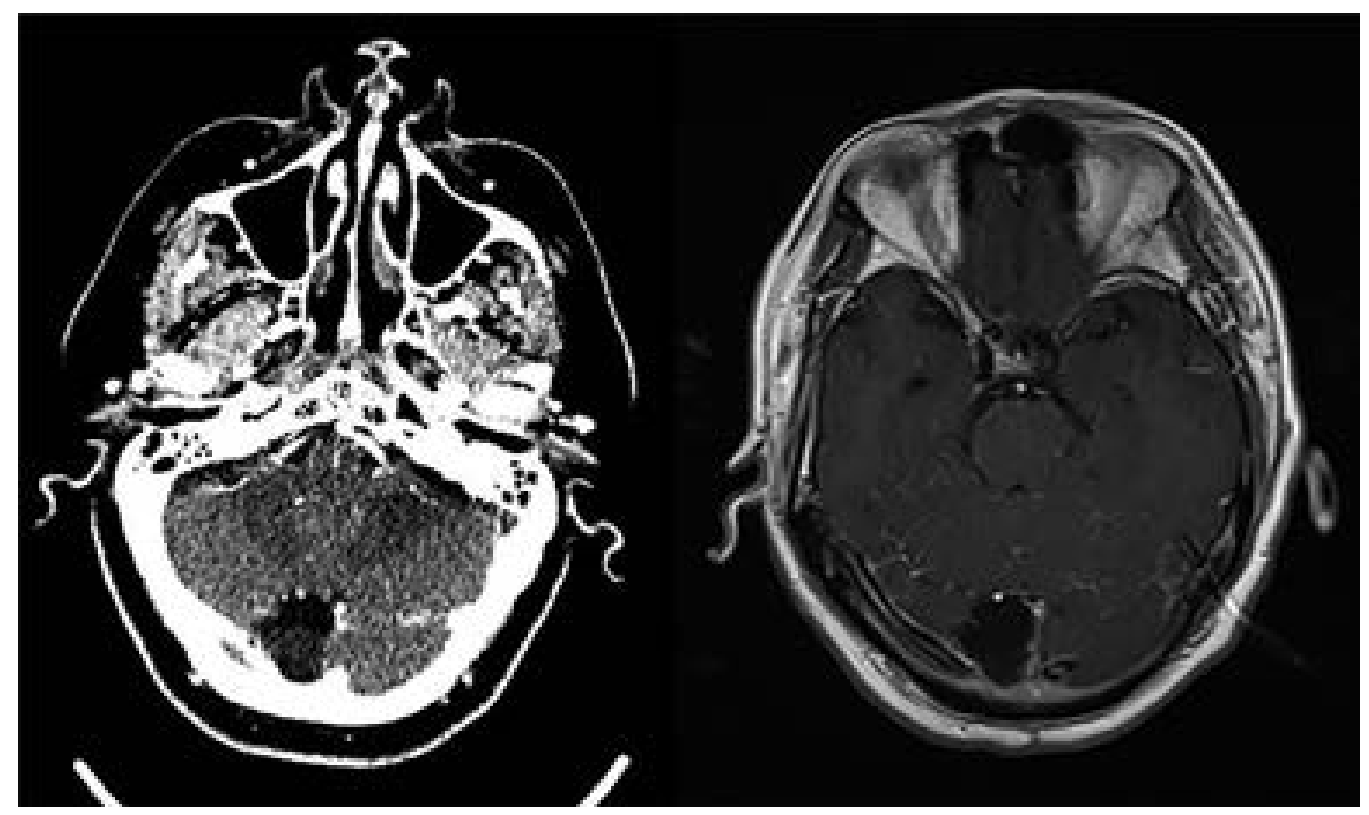

Figura 1. Tomografia espiral multicorte $(A)$ y resonancia magnética $(B)$ con imágenes compatibles con megacisterna magna en fosa posterior y ausencia de signos de hipertensión endocraneana 
en 0,66 ng/dL, confirmándose el diagnóstico adicional de hipotiroidismo.

Se realiza una punción lumbar control, al tercer día de antibioticoterapia, obteniéndose un líquido claro con 252 células $/ \mathrm{mm}^{3}$ (mononucleares $100 \%$ ), glucosa de $38 \mathrm{mg} / \mathrm{dL}$, proteínas en $116,5 \mathrm{mg} / \mathrm{dL}$, lactato en $0,7 \mathrm{mmol} / \mathrm{L}$ y ADA de $17 \mathrm{U} / \mathrm{L}$.

Se añade aciclovir $400 \mathrm{mg}$ cada 8 horas por lesiones sugerentes de infección por el virus Herpes Simple tipo 1, y Levotiroxina a $100 \mu \mathrm{g}$ cada 24 horas como tratamiento del hipotiroidismo de reciente diagnóstico.

El resultado del antibiograma del cultivo muestra SP con resistencia a bencilpenicilina (Concentración Mínima Inhibitoria $[\mathrm{CMl}]=2 \mu \mathrm{g} / \mathrm{ml})$, ceftriaxona $(\mathrm{CMl}=2 \mu \mathrm{g} / \mathrm{ml})$, eritromicina $(\mathrm{CMl}>8 \mu \mathrm{g} / \mathrm{ml})$ y sensible a linezolid $(\mathrm{CMl}<2 \mu \mathrm{g} / \mathrm{ml})$ y vancomicina $(\mathrm{CMl}<0,5 \mu \mathrm{g} / \mathrm{ml})$. El paciente cursó sin secuelas neurológica con buena evolución al seguimiento.

Se obtuvo el consentimiento del paciente para el reporte del caso.

\section{DISCUSIÓN}

En los últimos años, se han incrementado los reportes de cepas de SP con RC a nivel mundial ${ }^{(2-7)}$. En Latinoamérica el escenario no es distinto, un estudio de sensibilidad antibiótica y caracterización de serotipos de SP realizado en Perú por Castro et al, reportó la presencia de resistencia intermedia a ceftriaxona en el $16,7 \%$, de un total de 12 casos de pacientes con meningitis ${ }^{(8)}$. En adultos chilenos se ha reportado una resistencia a cefalosporinas de tercera generación del 1\%, menor a lo registrado en Perú, probablemente por diferencias en las políticas de uso racional de antibióticos o métodos para la determinación de la resistencia antibiótica ${ }^{(2)}$. Sin embargo, en Colombia, un estudio de vigilancia de serotipos y patrones de susceptibilidad antibiótica del SP con una duración aproximada de 11 años, determinó un 3,5\% de RC para infecciones invasivas diferentes a neumonía, ubicándose dentro de ellas a la meningitis aguda, siendo mayor a lo reportado en Chile. Estos hallazgos mostraron una tendencia de la RC a ser mayor en pacientes pediátricos ${ }^{\left({ }^{9}\right)}$.

En otros países como Cuba se han encontrado resultados similares al estudio colombiano. En un estudio realizado a partir de SP aislados de muestras de LCR, se encuentra una $\mathrm{RC}$ del $3,4 \%$ de un total de 237 aislamientos ${ }^{(10)}$. Si bien, la frecuencia de RC del SP aislados en LCR es relativamente baja y poco conocida en nuestro medio, es importante observar que se encuentra presente en diferentes países de Latinoamérica incluyendo al Perú. Como se ha reportado, hay la posibilidad de encontrar en pacientes que padecen enfermedad invasiva neumocócica cepas de SP con RC.

Sin embargo, tenemos que considerar la posibilidad de una mala respuesta a los beta-lactámicos debido a las propiedades farmacocinéticas presentadas por las cefalosporinas de tercera generación al momento de distribuirse a nivel de las meninges cuando la cepa no es completamente sensible ${ }^{(11)}$. Por tal motivo, sugerimos elegir con cautela la mejor opción terapéutica al momento de inicio de la antibioticoterapia empírica.

El antecedente de otalgia pudo haberse asociado a un cuadro de otitis media aguda; pero no se reportan síntomas adicionales como otorrea $u$ otros. Un estudio realizado en Argentina por Jimenez et al (12), encuentra que la otitis no es la causa subyacente más frecuente en adultos con meningitis aguda por SP, debido a que la causa más común son las fístulas del LCR que se asocian en su totalidad a meningitis a repetición, distinto a lo reportado en este caso. Sin embargo, el $97,5 \%$ de los patógenos aislados fueron sensibles a penicilinas. Por otro lado, los antecedentes quirúrgicos también podrían haber contribuido a la aparición de $\mathrm{RC}$ debido a que utilizan con frecuencia como profilaxis antibiótica a las cefalosporinas ${ }^{(13)}$.

Es importante contar con un antibiograma al momento de evaluar la terapéutica y evolución de un paciente con meningitis aguda. La CMI de ceftriaxona del SP aislado en nuestro paciente fue igual a $2 \mu \mathrm{g} / \mathrm{ml}$, compatible con la definición de RC, que define resistencia cuando el $\mathrm{CMI}$ es mayor o igual a $2 \mu \mathrm{g} / \mathrm{ml}^{(14)}$. Motivo por el que se continuó el esquema terapéutico con vancomicina con una respuesta clínicamente favorable, indicándonos la importancia de considerar a este antibiótico para el manejo empírico de la meningitis aguda.

Apoyando estos hallazgos, los estudios son concordantes en relación a la susceptibilidad antibiótica del SP a la vancomicina, mostrando una sensibilidad del $100 \%$ en los diferentes reportes que incluyen al Perú ${ }^{(8-10)}$. Es pertinente la reconsideración de los esquemas de tratamiento actuales para el manejo de la meningitis aguda. Sugerimos la inclusión de este medicamento, en combinación con ceftriaxona para el tratamiento empírico de la meningitis aguda. No se pudo determinar el serotipo de la bacteria, al no ser una prueba de uso rutinario, y tampoco por el poco impacto clínico que tendría sobre el manejo del paciente debido a la presencia del anti-biograma y la respuesta favorable al tratamiento. Sin embargo, se conoce que los serotipos más frecuentes encontrados en Perú son el 19F y 23F (8).

Entre los factores de riesgo para contraer enfermedades neumocócicas, tenemos a la edad, diabetes mellitus, enfermedad renal crónica, hipertensión arterial, entre otras ${ }^{(15)}$. Sin embargo, la enfermedad tiroidea no ha sido claramente 
descrita, a pesar de existir evidencia sobre la interacción entre la tiroides y el sistema inmune por su relación con el timo, órgano implicado en la producción de linfocitos ${ }^{(5)}$. Podríamos considerar al hipotiroidismo como un posible factor de riesgo para el desarrollo de infecciones en general, y en nuestro caso, estar posiblemente asociado a una enfermedad invasiva neumocócica. Probablemente la aparición de herpes labial estuvo asociada al estado de inmunosupresión sumado al estrés originado por el cuadro de meningitis aguda. Se reporta que puede aparecer en el $12 \%$ de pacientes con meningitis aguda por SP ${ }^{(12)}$.

La MM ha sido asociado a trastornos psiquiátricos como psicosis, esquizofrenia, trastorno obsesivo-compulsivo, entre otros, así como alteraciones en los parámetros de las funciones mentales de memoria y fluencia verbal, que no fueron observados en nuestro paciente ${ }^{(6,16,17)}$. No hay evidencia acerca de su asociación frente a un cuadro de meningitis aguda ni a sus complicaciones. Sin embargo, nuestro paciente no desarrollo complicaciones como hipertensión endocraneana. Al parecer, la MM podría haberse comportado como un factor protector frente a esta alteración, debido a un posible efecto sobre la circulación del LCR.
En conclusión, documentamos la presencia de SP con $\mathrm{RC}$ en el Perú, la cual probablemente se encuentre en aumento. El hipotiroidismo puede ser un factor de riesgo para desarrollar enfermedad invasiva neumocócica y la megacisterna magna un posible factor protector de complicaciones. Se recomienda agregar dentro del esquema antibiótico empírico a la vancomicina y más estudios de sensibilidad antibiótica y políticas estrictas para el uso racional de antibióticos en el país.

Agradecimientos. Al Dr. Fernando Mendo-Urbina por haber concedido los permisos y confianza en el equipo para hacer realidad el presente reporte.

Contribución de los autores: RPR, MTC, FMU, MSV y SCZDRA tuvieron la concepción y diseño del manuscrito, así como la recogida de datos, análisis e interpretación de los mismos, redacción, decisión y aprobación del manuscrito. JAGU realizó la revisión crítica del manuscrito.

Fuentes de financiamiento: La presente investigación no ha recibido ninguna beca específica de agencias de los sectores público, comercial, o sin ánimo de lucro.

Conflictos de interés: Los autores no declaran presentar conflictos de interés.

\section{REFERENCIAS BIBLIOGRÁFICAS}

1. García Vázquez E, Hernández Torres A Herrero Martínez JA, Gómez Gómez J. Protocolo terapéutico empírico de la meningitis infecciosa aguda. Med - Programa Form Médica Contin Acreditado. 2014;11(58):3448-51.

2. Hadad P, Gualda Á. Mala respuesta a ceftriaxona en adultos con meningitis por Streptococcus pneumoniae. Rev Chil Infectol. 2015;32(3):365-6.

3. Hauser $\mathrm{N}$, Cervera-Hernandez ME, Lonks J, Zaidi N. Rare but not forgotten: A case of meningitis due to ceftriaxoneresistant Streptococcus pneumoniae. IDCases. 2018;11:73.

4. Mendes RE, Biek D, Critchley IA, Farrell DJ, Sader HS, Jones RN. Decreased Ceftriaxone Susceptibility in Emerging (35B and 6C) and Persisting (19A) Streptococcus pneumoniae Serotypes in the United States, 2011-2012: Ceftaroline Remains Active In Vitro among $\beta$-Lactam Agents. Antimicrob Agents Chemother. 2014;58(8):4923-7.

5. Fabris N, Mocchegiani E, Provinciali M. Pituitary-thyroid axis and immune system: a reciprocal neuroendocrine-immune interaction. Horm Res. 1995;43(1-3):29-38.

6. Langarica M, Peralta V. Psicosis asociada a megacisterna magna. An Sist Sanit Navar. 2005;28(1):119-21.

7. Karlowsky JA, Adam HJ, Golden AR, Baxter MR, Nichol KA, Martin I, et al.
Antimicrobial susceptibility testing of invasive isolates of Streptococcus pneumoniae from Canadian patients: the SAVE study, 2011-15. J Antimicrob Chemother. 2018;73(suppl_7):vii5-11.

8. Castro JD, Siccha SM, Egoavil M, Chaparro E, Hernandez R, Silva W, et al. Resistencia antibiótica y distribución de serotipos en cepas neumocócicas invasivas en adultos hospitalizados en Lima, Perú. Rev Peru Med Exp Salud Pública. 2017;34(4):633-41.

9. Agudelo CI, Moreno J, Sanabria OM, Ovalle MV, Fabio JLD, Castañeda E. Streptococcus pneumoniae: evolución de los serotipos y los patrones de susceptibilidad antimicrobiana en aislamientos invasores en 11 años de vigilancia en Colombia (1994 -2004). Biomédica. 2006;26(2):234-49.

10. Toraño-Peraza G, Pías-Solis L, AbreuCapote M, Rodríguez-Ortega M, Dickinson-Meneses F, Varcárcel-Sánchez M. Serotipos y resistencia antimicrobiana de aislamientos meníngeos de Streptococcus pneumoniae. Cuba, 2007-2012. Vaccimonitor. 2014;23(3):117-23.

11. Kaplan SL. Management of pneumococcal meningitis. Pediatr Infect Dis J. 2002;21(6):589-91; discussion 613-614.

12. Jiménez Caballero PE, Serviá Candela M. Análisis descriptivo de las meningitis por Streptococcus pneumoniae en un hospital terciario. Neurol Argent. 2012;4(1):6-10.
13. IETSI. Guía de Práctica Clínica para la Profilaxis Antibiótica en Procedimientos Quirúrgicos. Guía en Versión Corta. Perú; 2017. (GPC N $\left.{ }^{\circ} 5\right)$.

14. M100Ed28 | Performance Standards for Antimicrobial Susceptibility Testing, 28th Edition [Internet]. Clinical \& Laboratory Standards Institute. [citado 4 de noviembre de 2018]. Disponible en: https://clsi. org/standards/products/microbiology/ documents $/ \mathrm{m} 100 /$

15. Lipsky BA, Boyko EJ, Inui TS, Koepsell TD. Risk factors for acquiring pneumococcal infections. Arch Intern Med. 1986;146(11):2179-85

16. Pandurangi S, Pandurangi A, Matkar A, Shetty N, Patil P. Psychiatric manifestations associated with mega cisterna magna. J Neuropsychiatry Clin Neurosci. 2014;26(2):169-71.

17. Zimmer EZ, Lowenstein L, Bronshtein M, Goldsher D, Aharon-Peretz J. Clinical significance of isolated mega cisterna magna. Arch Gynecol Obstet. 2007;276(5):487-90.

Correspondencia: Rafael Pichardo-Rodriguez Correo:rafael_martin1352@hotmail.com Dirección: Av. Javier Prado Este 3028, San Borja 15037

Celular: 941066554 